\section{The Effects of Chiropractic Spinal Manipulation on Uri- nary Incontinence in Patients with Low Back Pain and Ra- diculopathy: A Retrospective Case Series Report}

\author{
Mohsen Khamessipour ${ }^{1}$ and Michael Hall ${ }^{*}$ \\ ${ }^{1}$ Department of Chiropractic Clinical Neurology, Research and Science \\ Committee, IRCA, Tehran, Iran
}

${ }^{2}$ Department of Clinical Neurology and Clinical Sciences, Parker University, Dallas, Texas, USA

\begin{abstract}
Urinary Incontinence (UI) is a common dysfunction among patients specially women with low back pain and radiculopathy. The pelvic symptomatology that these patients demonstrate may be attributable to lower sacral nerve root compression that is most commonly the result of Lumbar Disc Herniation (LDH).

Objective: The effects of chiropractic spinal manipulation on urinary incontinence in patients with low back pain and radiculopathy are the focus of this report.

Methods: A total of 119 patients' data (108 female, 11 male, mean ages $59.7 \pm 11.6$ years) with low back pain and leg pain that all admit to have urinary control problems were reviewed. Lumbar or Lumbosacral Disc Herniation (LDH) with radiculopathy diagnosis was confirmed with complete chiropractic, orthopedic and neurologic evaluation, MRI of the lumbar and lumbosacral regions and electrodiagnostic tests (Electromyogram and Nerve Conduction Studies EMG/NCS) of the lower limbs. Chiropractic manipulative therapy involving high-velocity, low-amplitude manipulation and flexion distraction manipulation was performed.
\end{abstract}

*Corresponding author: Michael Hall, Department of Clinical Neurology and Clinical Sciences, Parker University, Dallas, Texas, USA, Tel: +1 9492854415; E-mail: michael.hall6@life.edu; michael.hall@life.edu

Citation: Khamessipour M, Hall M (2017) The Effects of Chiropractic Spinal Manipulation on Urinary Incontinence in Patients with Low Back Pain and Radiculopathy: A Retrospective Case Series Report. J Altern Complement Integr Med 3: 042.

Received: September 15, 2017; Accepted: October 27, 2017; Published: November 13, 2017
Results: After 1-8 weeks of chiropractic treatment, the overall urinary frequency and incontinence was significantly reduced from 4.2 to 1.3 episodes $(P<0.001)$.

Conclusion: Chiropractic spinal manipulation exerts positive physiological responses in patients with LBP and radiculopathy and UI. Therefore, chiropractic treatment may be considered as a conservative and effective means of treating pelvic disorders secondary to lower sacral nerve root compression.

\section{Introduction}

Back pain is a highly prevalent musculoskeletal condition. Specifically, Low Back Pain (LBP) is the most common type of pain reported by US adults, with one in four adults reporting the experience of LBP in the past 3 months. Furthermore, a reported $70-85 \%$ of adults will experience an episode of LBP at some point in their lifetime [1]. Low back pain has been linked to work-family imbalance, exposure to a hostile work environment, job insecurity, long work hours and certain occupation groups [2].

Low Back Pain (LBP) and Urinary Incontinence (UI) are prevalent among elderly individuals especially in women. LBP and UI are conditions that strongly affect functioning in the elderly and hinder the performance of everyday activities, thus causing physical and emotional distress, incurring high socioeconomic costs, restricting social participation, and decreasing the quality of life [3]. There are large variations in the severity and impact of incontinence. Its severity, frequency, and predictability all need to be considered when evaluating its effects on patients.

The two main types of incontinence are stress (effort) and urge incontinence. The term mixed incontinence denotes the concomitant appearance of stress and urge incontinence. The International Continence Society, ICS, has published (2001) definitions at symptom level for the different forms of incontinence in adults. Stress urinary incontinence is the complaint of involuntary leakage on effort or exertion, or on sneezing or coughing. Urge urinary incontinence is the complaint of involuntary leakage accompanied by or immediately preceded by urgency. Mixed urinary incontinence is the complaint of involuntary leakage associated with urgency and also with exertion, effort, sneezing or coughing.

Other pertinent symptoms and syndromes suggestive of lower urinary tract dysfunction which may or may not be accompanied by urinary leakage are Nocturia which is the complaint that the individual has to wake at night one or more times to void, and urgency which is the complaint of a sudden compelling desire to pass urine which is difficult to defer. Overactive Bladder syndrome $(\mathrm{OAB})$ is defined as urgency with or without urge incontinence, usually combined with frequency and nocturia. In men, $\mathrm{OAB}$ is frequently associated with benign prostate hyperplasia. Urgency-frequency syndrome is the complaint of urgency without involuntary leakage of urine. It may be part of the OAB syndrome [4].

In young women, the prevalence of incontinence is usually low, but prevalence peaks around menopause, with a steady rise there-after 
into later life [5]. In women, moderate and severe UI have a prevalence ranging from about $3 \%$ to $17 \%$. Severe incontinence has a low prevalence in young women, but rapidly increases at ages 70 through 80. In men, the prevalence of incontinence is much lower than in women, about $3 \%$ to $11 \%$.

Several studies have shown an association between back pain and UI $[1,6,7]$. In one study a significant association between CBP and stress UI was found, and authors concluded that it is important for all trunk muscles, including the pelvic floor muscles, to function in coordination with one another for postural control and for prevention of pain and dysfunction. Therefore, it may be possible that the management of chronic back pain includes assessment of and treatment for the pelvic floor muscles [1]. A cross-sectional study of women only by Smith et al., found a relationship between continence disorders and back pain [8]. Also the association between UI and LBP and radiculopathy in women was investigated and authors concluded that LBP should not be regarded as a predisposing factor for urinary incontinence; however, radiculopathy has a statistically positive correlation between overall incontinence and urge incontinence [9]. In another study investigating UI in women with low back pain it was found that UI was reported by $78 \%$ of women with LBP, and that suffering from LBP and inability to interrupt the urine flow increased the risk for UI and the prevalence of UI was greatly increased among women with LBP [7]. Finkelstein et al., reported a strong association between "back problems" and UI in both men and women [7]. In addition, Kim et al., found women with greater UI severity also have a higher perceived severity of LBP and LBP perceived disability [10]. Lastly, Eliasson et al., surveyed women who were receiving physical therapy for LBP and reported 78\% of these women also reported UI [7].

When the association between chronic back pain and urinary incontinence in women was investigated, they concluded, "Women who report Chronic Back Pain (CBP) have an increased odds of having stress UI. Therefore, clinicians must consider this association and the relationship of relevant trunk muscles, including pelvic floor musculature, in patients presenting with CBP and UI" [11].

Despite a focus on LBP management by doctors of chiropractic, limited published accounts detail clinical outcomes with chiropractic management of LBP among patients with UI.

\section{Methods}

\section{Design}

This study was a retrospective case series of patient records. Authors collected retrospective data from the records of the LBP with Radiculopathy patients seen in their private chiropractic clinics who were treated with SMT including FD. Informed consent was obtained from all patients who met the inclusion criteria for this study.

\section{Sample}

Data from 1367 patients with a chief complaint of LBP with leg pain were reviewed; 119 cases had UI (8.7\%) simultaneously. Data from these 119 patients (108 female, 11 male, mean ages $59.7 \pm 11.6$ years) with low back and leg pain that all admit to have urinary control problems were reviewed (Figure 1).

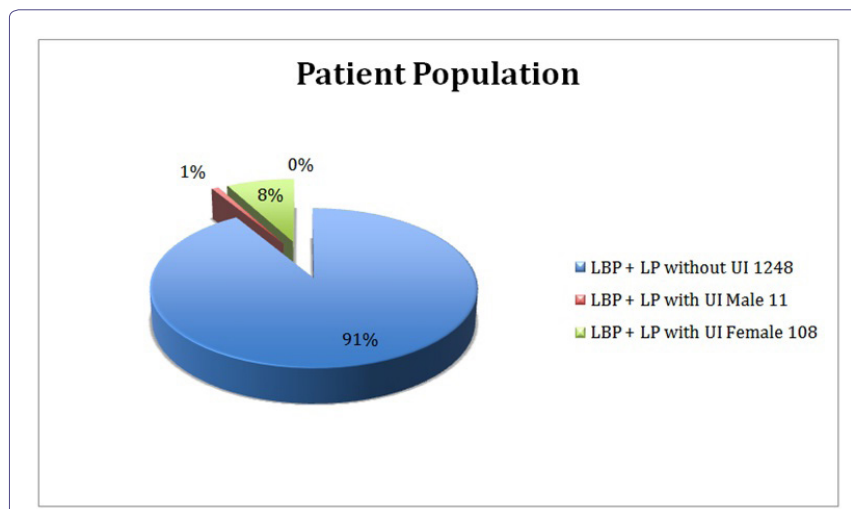

Figure 1: Patient Population.Prevalence of LBP, LP and UI.

All patients selected had a history of UI between 2 months to 12 years. They also had chronic low back and/or pelvic pain and leg pain of more than 2 years simultaneously.

Inclusion criteria included presence of lumbar (L3/4, L4/5) and or lumbo-sacral disc (L5/S1) herniation compromising neural tissues confirmed by MRI studies and leg pain that was confirmed as radiculopathy by Electromyography (EMG), Nerve Conduction Studies (NCS) and neurological findings such as altered reflexes, decreased sensation and reduced lower extremity muscle strength found during the examination procedures.

Exclusion criteria included presence of any severe neurological disorder such as neurodegenerative disease, cauda equina syndrome, CNS tumors, or any urological disorders such as bladder or sphincter dysfunction.

Lumbosacral disc pathology with radiculopathy diagnosis was confirmed with complete chiropractic evaluation, which involves variety of methods to determine the spinal segments that require chiropractic treatments, including but not limited to static and motion palpation techniques determining spinal segments that are hypo mobile (restricted in their movement) or fixated, orthopedic and neurologic evaluation, MRI of the lumbosacral region, and EMG/NCS of the lower limbs. EMG/NCS confirmed the diagnosis of radiculopathy in all patients in this study. Electrodiagnostic testing is of great value in confirming the diagnosis of suspected radiculopathy or sciatic neuropathy and assessing the potential for recovery of nerve function [12]. The MRI studies of all patients in this study demonstrated the presence of lumbar (L3/4, L4/5) or lumbo-sacral (L5/S1) disc herniation, with evidence of neural tissue compromise.

\section{Chiropractic Treatment}

\section{Methods and Frequency}

Chiropractic manipulative therapy treatments for the low back region, alone or in combination, included Flexion Distraction (FD) and Spinal Manipulative Therapy (SMT) were utilized. The number of treatments for each patient was determined by multiple factors including nature of their presentation. The mean number of treatments was 7.5. The typical course of care consisted of two to three treatments a week, with a reevaluation and review of updated outcome measures after every treatment session. 
The complexity of various patient presentations likely influenced practice patterns with regard to manual treatment selection. FD or spinal mobilization may have been used in favor of SMT in the presence of underlying bone weakening disorders (osteoporosis), spondylolysis, or surgical fusion or hardware, where the provider deemed a more gentle approach appropriate.

Chiropractic treatment effectiveness for spinal disc pathology has also been demonstrated by several studies. Chiropractic treatments accelerate the recovery time for both acute and chronic lumbar disc herniation when compared to the natural history of recovery. In an attempt to update a previously published evidence-based practice guideline on chiropractic management of low back pain authors concluded that the evidence supports that doctors of chiropractic are well suited to diagnose, treat, co-manage and manage the treatment of patients with low back pain disorders [13]. Until very recently the database on effectiveness of chiropractic treatments for disc herniation has been poor, but in a study by Leemann et al., which follows on the heels of several other recent papers, demonstrates the effectiveness of chiropractic treatments in promoting recovery based on disability scores, numerical pain rating scores and patient global impression of change [14]. In a case report the author concludes that chiropractic treatment may be an effective means of treating pelvic disorders secondary to lower sacral nerve root compression [15]. In another study Cox et al., found that greater than $50 \%$ pain relief following chiropractic distraction spinal manipulation was seen in $81 \%$ of postsurgical patients receiving a mean of 11 visits over a 49-day period of active care [16]

SMT is generally a safe intervention, most commonly associated with only benign, temporary side effects including local soreness which typically does not interfere with regular activity [17]. An estimate of the risk of spinal manipulation causing a clinically worsened disc herniation or Cauda Equina Syndrome (CES) in a patient presenting with LDH is calculated from published data to be less than 1 in 3.7 million. Cauda equina syndrome seems to be a very rare complication of spinal manipulations. Only few cases, in fact, were referred in literature in the past decades [18].

The apparent safety of spinal manipulation, especially when compared with other "medically accepted" treatments for Lumbar Disc Herniation (LDH), should stimulate its use in the conservative treatment plan of LDH [19]. A systematic review of randomized controlled trials of the most common interventions for chronic LBP showed that there is strong evidence that manipulation is more effective than a placebo treatment for chronic LBP [20].

FD is a manual procedure designed to produce continuous passive spinal motion in a prone posture with elements of traction assisted by manual pressure applied to the low back region [21]. SMT is a form of manually assisted passive motion involving a high-velocity-low-amplitude thrust to the spine that is generally applied with the patient positioned in a lateral decubitus posture [22]. Licensed doctors of chiropractic provided treatments. Our chiropractic intervention was multi-modal and involved chiropractic SMT and FD treatments for the low back region and LDH. Instructions for therapeutic home exercise and stretches tailored to the patient's specific condition and presentation to strengthen the spinal musculature and increase flexibility were prescribed. However, pelvic floor exercises specific for treating UI were not prescribed since treating UI in patients under our care for low back pain and radiculopathy was not part of the overall treatment plan.

\section{Clinical Outcomes}

The primary outcome measure for this analysis was the frequency of nocturia and instances of leakage or Incontinence Episodes (IE) reported by the patients during each office visit. For this study a Minimum Clinically Important Difference (MCID) was set as 30\% improvement from baseline. The primary outcome measure for this study was purely subjective and there were not any objective outcome measures used in this trial, which is a limitation by itself.

The concept of Minimum Important Difference (MID) represents the magnitude of benefit for which randomized controlled trials should be powered in order to minimize type 1 and type 2 errors (false positives and false negatives). Likewise, they can be used as clinical markers of improvement, as well as gauges for interpreting future studies. There are two methods of determining MID, anchor-based and distribution-based [23]. MICD values are population-based and an individual patient's perception may not correlate. Statistically significant changes (improvements) in International Consultation on Incontinence Questionnaire ICIQ scores that meet these time-specific thresholds can be considered clinically important [24].

The MICD estimates the minimum degree of change in score correlating with patient perception of improvement. There is no "gold standard" methodology of estimating the MCID or achieving the meaningfulness of clinical trial results based on patient reported outcomes [25]. The difference in (ICIQ) scores of patients with a $>75 \%$ reduction in IE to those with no change was compared. The accepted MICD as the difference between "better" ( $>25 \%$ decrease in IE ) and no change (change in any direction between 0 and $24 \%$ in IE) was defined. The MICD was the difference between "better" and "no change" [24].

\section{Results}

After 1-8 weeks of chiropractic treatment, the overall urinary frequency and Incontinence Episodes (IE) in this study was significantly reduced from 4.2 to 1.3 episodes $(\mathrm{P}<0.001)$. The mean number of treatments was 7.5. 6 (5.04\%) patients did not meet the MCID. 36 (30.25\%) patients met the MCID and 77 (64.74\%) patients' UI symptoms were completely resolved (Figure 2). Periodic follow-up examinations indicate that for each participant in this case-series report, the improvements of UI remained stable and persisted over time. There were no reports of patients who showed deterioration of UI in this study.

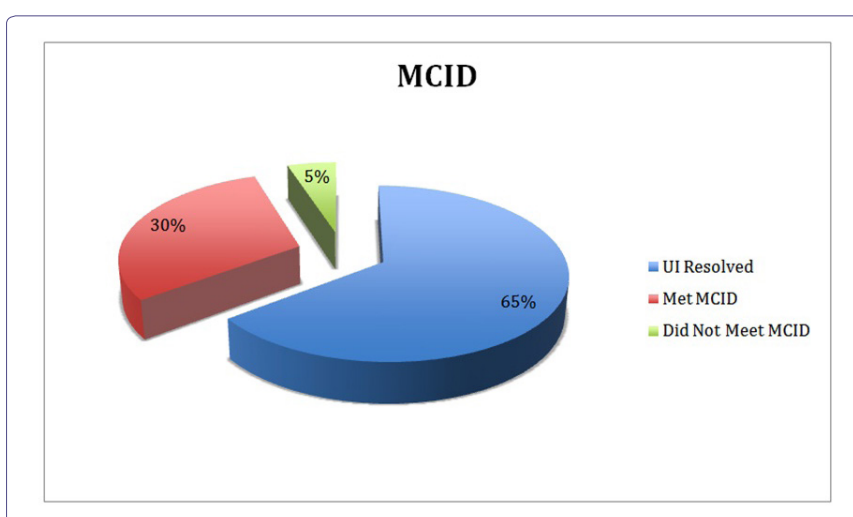

Figure 2: Minimum Clinically Important Difference (MCID). 


\section{Discussion}

Several theories have been put forth in attempts to explain the possible mechanisms by which patients presumed to be suffering from any of a variety of internal organ diseases are occasionally found to respond quickly and dramatically to therapies delivered to purely somatic structures (e.g., spinal manipulation). The rapidly growing and highly convincing body of knowledge concerning the general concept of somatic visceral disease simulation provides a scientifically sound alternative explanation for the apparent effectiveness of a variety of somatic therapeutic interventions in patients presumed to be suffering from true visceral disease [26]. This somato-visceral disease connection can create signs and symptoms that are virtually indistinguishable with respect to their somatic vs. visceral etiologies. Furthermore, it is not unreasonable that this somatic visceral-disease mimicry could very well account for the "cures" of presumed organ disease that have been observed over the years in response to various somatic therapies (e.g., spinal manipulation, acupuncture, Rolfing, Qi Gong, etc.,) and may represent a common phenomenon that has led to "holistic" health care claims on the part of such clinical disciplines [3,26].

The unusual association of low back pain and leg pain, with urinary incontinence should be brought to the attention of clinicians, in the search for neurologic mechanisms to explain the phenomenon. A rare association between severe low back pain and urgency incontinence of urine, not explained on the basis of any conventional neurologic or genito-urinary pathology, should be recognized [27]. Many large studies reporting on the prevalence of incontinence in a specific population do not distinguish between the different types or causes of incontinence. Neurological conditions including both the central (CNS) and Peripheral Nervous System (PNS) disorders should always be considered as a possible etiology.

The CNS is greatly influenced by somatosensory input. Numerous, seemingly unrelated symptoms can be generated when nociceptive input is enhanced and mechanoreceptive input is reduced. The neuropathophysiological effects of joint complex dysfunction resulting in dysafferentation from mechanoreceptors could explain why so many seemingly bizarre symptoms respond to chiropractic care when the proper afferentation from the mechanoreceptors is restored [28].

Research has shown that sensory input from paraspinal tissues can evoke visceral reflexes affecting the sympathetic nervous system and may alter end-organ function. In general, non-noxious paraspinal sensory input appears to have an inhibitory effect on sympathetic outflow, whereas noxious input appears to have an excitatory effect. The data are provocative, indicating that neural input from axial tissues can evoke somatovisceral reflexes [29].

Additionally Sato and Swenson investigated the effects of mechanical stimulation of the spine on blood pressure, heart rate and the activity of selected sympathetic nerves (renal and adrenal) were examined in alpha-chloralose/urethane anesthetized rats and concluded that their present study has demonstrated potent somatovisceral reflexes from mechanical stimulation of the spinal column [30].

Organic causes of UI include bladder dysfunction, sphincter dysfunction, or a combination of both. There have been a number of large surveys where questionnaires are the primary tool for evaluating the presence of incontinence. In these studies it is often difficult if not impossible to determine the cause of incontinence [5]. Vania $\mathrm{F}$ demonstrated abdominal and pelvic floor muscle reduced activation as a cause of UI [3]. A cross-sectional study found that women who report Chronic Back Pain (CBP) have an increased odds of having stress UI. Therefore clinicians must consider this association and the relationship of relevant trunk muscles, including pelvic floor musculature, in patients presenting with CBP and UI [1].

Spinal cord nuclei supplying the vesicourethral smooth muscle and rhabdosphincter are in the lumbosacral region [31]. The results of recent electrophysiological investigations indicate that many patients with urological, bowel or anorectal dysfunction demonstrate evidence of denervation neuropathy in muscles innervated by the branches of the pudendal nerve, which derives its fibers from the ventral rami of the second, third and fourth sacral nerves (S2, S3, S4) [32].

The urologic manifestations of neurodegenerative disorders, including Parkinson Disease (PD), multisystem atrophy and Huntington chorea, have been studied by several groups. Patients with multisystem atrophy and autonomic failure have considerable difficulty with incontinence. Sphincter EMG of these patients has shown abnormal motor units and increased motor unit durations. Patients with multisystem atrophy and Shy-Drager syndrome are known to have cell loss in the motor nuclei at S2-S4; this finding has led to increased speculation concerning the autonomic properties of these neurons [31].

Spinal cord lesions produce various types of voiding dysfunction, depending on the level of the neuro-axis involved. Central disc herniation is particularly important since acute central herniation of a disc at L5-S1 may be a neurosurgical emergency when the patient comes in with sudden onset of UI. This is because the nerve roots of the Cauda Equina that supply the detrusor travel medially. The sensory inputs from the bladder are not affected. In central herniation, patients usually experience no sciatic radiation of pain [31].

Spinal stenosis in the lumbosacral region, as it begins producing symptoms, usually results in intermittent episodes of urinary retention as a manifestation of intermittent claudication of the conus or cauda equina. Stenosis in the cervical region is more likely to produce longtract involvement with bladder sphincter dyssynergia; that is, contraction of the bladder is accompanied by abnormal contraction of the sphincteric mechanism [31].

Cervical spinal cord disorders may also show its neurological manifestations in the pelvic region. There is tantalizing new evidence suggesting that muscle spindles in cervical paraspinal muscles may in fact be capable of eliciting somato-autonomic reflexes [33]. Other studies have investigated the relationship between the spinal cord stimulation and the resultant autonomic response. A study of spinovisceral reflexes reported responses of bladder motility to noxious spinal stimulation [34]. Additionally, there is recent evidence from studies in conscious humans that mechanical stimulation of the neck may influence cardiovascular function [35]. Carrick FR concluded that cervical manipulation activates specific neurological pathways. Manipulation of the cervical spine may be associated with an increase or a decrease in brain function depending upon the side of the manipulation and the cortical hemisphericity of a patient [36].

Mechanoreceptors are thought to have roles in maintaining muscle tone and reflexes.

Appropriate mechanoreceptor afferent input is required by the cerebral cortex to perform a host of conscious and subconscious motor 
functions. If afferent signals were eliminated, the cerebrum would be incapable of functioning in a conscious manner and would actually approach a permanent state of coma. Mechanoreceptor input is actually needed to help us function as humans. Divergence in the totality of sensory input could ultimately result in pathology, or symptoms of pathology, in seemingly unrelated tissues and organs.

Mechanoreceptors are present in the spinal joint complex including the intervertebral disc, and were found in the outer 2-3 lamellae of the human intervertebral disc and anterior longitudinal ligament. Investigation of the occurrence and morphology of mechanoreceptors in human and intervertebral discs and longitudinal ligaments conclude that the presence of mechanoreceptors in the intervertebral disc and longitudinal ligament can have physiologic and clinical implications [37]. Mechanoreceptive information reaches numerous centers in the CNS. Consequently, a reduction in mechanoreceptor input caused by joint complex dysfunction has the potential to promote numerous symptoms that could mimic lesions of the vestibular nuclei, cerebellum, cerebral cortex and basal ganglia. Although much more research is still needed in this area, evidence exists to support this contention [28]. Also reduced mechanoreception may also impact the non-motor functions of the cerebellum. In 1978, Watson explained how traditional concepts of cerebellar physiology emphasize motor control functions; however, he also points out that an emerging body of literature demonstrates a relationship between the cerebellum and psychological processes [38].

Mechanoreceptor afferents (A-beta fibers) influence the nervous system in many ways. Reduced mechanoreceptive activity will enhance the nociceptive input associated with joint complex dysfunction. At the spinal cord level, mechanoreceptor input can inhibit nociception (The Gate Theory) as well as reduction in sympathetic hyperactivity. Also, nociceptive stimulation of autonomic centers in the brainstem, particularly the medulla causes autonomic abnormalities. Many neuroendocrine abnormalities can also occur in response to nociceptive input. This creates complex patterns of signs and symptoms that can often be virtually identical to and, therefore, easily mistaken for those induced by primary visceral disease.

Effects of dysafferentation to higher centers in the nervous system from the spinal nerve roots may be more evident in this study where it was concluded that the pons can initiate bladder contractions and coordinated bladder-sphincter activity, but that afferent feedback (via the dorsal roots) is needed to maintain the large amplitude micturition contractions [39].

Reduced joint mobility results in less mechanoreceptor activation. Only $9 \%$ of the fibers in the medial articular nerve and $26 \%$ in the posterior articular nerve are mechanoreceptive. This low percentage of mechanoreceptive afferents suggests that maintaining joint mobility is crucial for proper signaling and afferent input to the CNS.

The symptoms generated by joint complex dysfunction, such as pain, nausea and vertigo, are probably caused by increased nociceptive input and/or reduced mechanoreceptive input. Joint complex dysfunction should be included in the differential diagnosis of pain and visceral symptoms because joint complex dysfunction can often generate symptoms which are similar to those produced by true visceral disease [28].

The mean number of treatments was relatively low for this sample at 7.5 visits. The patients treated in this current report achieved a clinically and statistically significant change in UI symptoms over what appears to be a relatively short course of care.

We believe components that may have been active in altering UI frequency are increased afferentation from mechanoreceptor activity as well as restoration of normal neuronal function by reducing lumbar and sacral nerve roots irritation and compression caused by LDH following SMT.

Nonmedical treatment of incontinence involves the use of pelvic floor muscle training, Tibial nerve stimulation, biofeedback, pessaries, bladder retraining, and sometimes intermittent catheterization. For mild stress incontinence, Kegel exercises may increase pelvic muscle tone enough to correct the problem [31].

Changes in motor control following spinal manipulation were investigated and found increases in strength following spinal manipulation were due to descending cortical drive and could not be explained by changes at the level of the spinal cord. Spinal manipulation may therefore be indicated for the patients who have lost tonus of their muscle and/or are recovering from muscle degrading dysfunctions such as stroke and/or orthopedic operations [40]. Many studies show that chiropractic adjustments result in changes to sensorimotor integration within the central nervous system [41]. Cortical effects of chiropractic spinal manipulation have been well documented. Investigators were able to demonstrate that a single session of spinal manipulation of dysfunctional segments in subclinical pain patients alters somatosensory processing at the cortical level, particularly within the prefrontal cortex [42].

De Almeida and his colleagues showed that SMT of the sacrum was associated with an increase in phasic perineal contraction and of basal perineal tonus in women who had no associated osteoarticular diseases [43]. Therefore, chiropractic management of pelvic dysfunction, especially UI, should also be considered when the patient meets the inclusion criteria set forth by this study and non-surgical or conservative treatment options are prescribed. Other studies show that spinal manipulation may alter sensorimotor integration. These findings may help elucidate mechanisms responsible for the effective relief of pain and restoration of functional ability documented after spinal manipulation [44].

\section{Conclusion}

The increasing prevalence of UI among patients with LBP and radiculopathy should lend support to investigating conservative forms of management that have demonstrated effectiveness. Despite comorbidity, chiropractic clinical outcomes in terms of improvement percentage from baseline for these patients were clinically meaningful and statistically significant.

Although Cauda Equina Syndrome (CES) seems to be a very rare complication of spinal manipulations but as a potential complication of lumbar spinal manipulation, chiropractors must be well equipped to recognize and immediately refer those with CES.

It is conceivable to the authors that chiropractic spinal manipulation exerts positive physiological responses in these patients. The results of this study are very promising with 113 (94.95\%) patients reporting improvements. These results are positive enough to warrant the need for a large study with medical participation (Urologists). Future investigations into clinical outcomes and the duration of courses of chiropractic care are warranted, with a broader representation of clinic settings and providers. 
This study adds to the understanding of chiropractic clinical outcomes and encourages further chiropractic research. Future studies may look at:

- The segmental and supra-segmental influences from spinal manipulative procedures and UI

- Association of joint complex dysfunction induced mechanoreceptor dysafferentation and resulting aberrant neuroplastic changes in the nervous system

- Compression or irritation of neural tissue occurring at the lower lumbar levels compromising neuronal function of the sacral nerves need to be further investigated for its contribution to pelvic conditions that may be occurring simultaneously

While the accuracy of the data were ensured, study limitations still include those inherent to the nature of retrospective design.

\section{References}

1. Bush HM, Pagorek S, Kuperstein J, Guo J, Ballert KN, et al. (2013) The Association of Chronic Back Pain and Stress Urinary Incontinence: A Cross-Sectional Study. J Womens Health Phys Therap 37: 11-18.

2. Yang H, Haldeman S, Lu ML, Baker D (2016) Low Back Pain Prevalence and Related Workplace Psychosocial Risk Factors: A Study Using Data From the 2010 National Health Interview Survey. J Manipulative Physiol Ther 39: 459-472.

3. Figueiredo VF, Amorim JSC, Pereira AM, Ferreira PH, Pereira LSM (2015) Associations between low back pain, urinary incontinence, and abdominal muscle recruitment as assessed via ultrasonography in the elderly. Braz J Phys Ther 19: 70-76.

4. European Medicines Agency (2013) Guideline on the clinical investigation of medicinal products for the treatment of urinary incontinence. Science Medicines Health, European Medicines Agency, London, UK.

5. Nitti VW (2001) The Prevalence of Urinary Incontinence. Rev Urol 3: 2-6.

6. Finkelstein MM (2002) Medical conditions, medications, and urinary incontinence. Analysis of a population-based survey. Can Fam Physician 48 : 96-101.

7. Eliasson K, Elfving B, Nordgren B, Mattsson E (2008) Urinary incontinence in women with low back pain. Man Ther 13: 206-212.

8. Smith MD, Russell A, Hodges PW (2006) Disorders of breathing and continence have a stronger association with back pain than obesity and physical activity. Aust J Physiother 52: 11-16.

9. Teodorczyk-Injeyan JA, McGregor M, Triano JJ, Injeyan HS (2017) Elevated Production of Nociceptive CC-chemokines and sE-selectin in Patients with Low Back Pain and the Effects of Spinal Manipulation: A Non-randomized Clinical Trial. Clin J Pain.

10. Kim JS, Kim SY, Oh DW, Choi JD (2010) Correlation between the Severity of Female Urinary Incontinence and Concomitant Morbidities: A Multi-Center Cross-Sectional Clinical Study. Int Neurourol J 14: 220-226.

11. Dunn AS, Green BN, Formolo LR, Chicoine D (2011) Retrospective case series of clinical outcomes associated with chiropractic management for veterans with low back pain. J Rehabil Res Dev 48: 927-934.

12. Distad BJ, Weiss MD (2013) Clinical and Electrodiagnostic Features of Sciatic Neuropathies. Phys Med Rehabil Clin N Am 24: 107-120.

13. Globe G, Farabaugh RJ, Hawk C, Morris CE, Baker G, et al. (2016) Clinical Practice Guideline: Chiropractic Care for Low Back Pain. J Manipulative Physiol Ther 39: 1-22.
14. Leemann S, Peterson CK, Schmid C, Anklin B, Humphreys BK (2014) Outcomes of acute and chronic patients with magnetic resonance imaging-confirmed symptomatic lumbar disc herniations receiving high-velocity, low-amplitude, spinal manipulative therapy: a prospective observational cohort study with one-year follow-up. J Manipulative Physiol Ther 37: 155-163.

15. Browning JE (1987) Pelvic pain and organic dysfunction in a patient with low back pain: response to distractive manipulation: a case presentation. $\mathrm{J}$ Manipulative Physiol Ther 10: 116-121.

16. Gudavalli MR, Olding K, Joachim G, Cox JM (2016) Chiropractic Distraction Spinal Manipulation on Postsurgical Continued Low Back and Radicular Pain Patients: A Retrospective Case Series. J Chiropr Med 15: 121-128.

17. Bronfort G, Haas M, Evans R, Kawchuk G, Dagenais S (2008) Evidence-informed management of chronic low back pain with spinal manipulation and mobilization. Spine J 8: 213-225.

18. Tamburrelli FC, Genitiempo M, Logroscio CA (2011) Cauda equina syndrome and spine manipulation: case report and review of the literature. Eur Spine J 20: 128-131.

19. Oliphant D (2004) Safety of spinal manipulation in the treatment of lumbar disk herniations: a systematic review and risk assessment. J Manipulative Physiol Ther 27: 197-210.

20. van Tulder MW, Koes BW, Bouter LM (1997) Conservative treatment of acute and chronic nonspecific low back pain. A systematic review of randomized controlled trials of the most common interventions. Spine (Phila $\mathrm{Pa}$ 1976) 22: 2128-2156.

21. Triano JJ, McGregor M, Skogsbergh DR (1997) Use of chiropractic manipulation in lumbar rehabilitation. J Rehabil Res Dev 34: 394-404.

22. Herzog W (2000) Clinical Biomechanics of Spinal Manipulation. Churchill Livingstone, London, UK.

23. Dyer KY, Xu Y, Brubaker L, Nygaard I, Markland A, et al. (2011) Urinary Incontinence Treatment Network (UITN). Minimum important difference for validated instruments in women with urge incontinence. Neurourol Urodyn 30: 1319-1324.

24. https://www.ics.org/Abstracts/Publish/180/000018.pdf

25. Brozek JL, Guyatt GH, Schünemann HJ (2006) How a well-grounded minimal important difference can enhance transparency of labeling claims and improve interpretation of a patient reported outcome measure. Health Qual Life Outcomes 4: 69.

26. Nansel D, Szlazak M (1995) Somatic dysfunction and the phenomenon of visceral disease simulation: a probable explanation for the apparent effectiveness of somatic therapy in patients presumed to be suffering from true visceral disease. J Manipulative Physiol Ther 18: 379-397.

27. Eisenstein SM, Engelbrecht DJ, el Masry WS (1976) Low back pain and urinary incontinence. A hypothetical relationship. Spine 19: 1148-1152.

28. Seaman DR, Winterstein JF (1998) Dysafferentation: a novel term to describe the neuropathophysiological effects of joint complex dysfunction. A look at likely mechanisms of symptom generation. J Manipulative Physiol Ther 21: 267-280.

29. Pickar JG (2002) Neurophysiological effects of spinal manipulation. Spine J 2: $357-371$

30. Sato A, Swenson RS (1984) Sympathetic nervous system response to mechanical stress of the spinal column in rats. J Manipulative Physiol Ther 7: 141-147.

31. Dyro FM, Egan RA (2014) Urologic Management in Neurologic Disease. Medscape.

32. Browning JE (1989) Chiropractic distractive decompression in treating pelvic pain and multiple system pelvic organic dysfunctions. J Manipulative Physiol Ther 12: 265-274. 
33. Bolton PS, Kerman IA, Woodring SF, Yates BJ (1998) Influences of neck afferents on sympathetic and respiratory nerve activity. Brain Res Bull 47: 413-419.

34. Budgell B, Hotta H, Sato A (1998) Reflex responses of bladder motility after stimulation of interspinous tissues in the anesthetized rat. J Manipulative Physiol Ther 21: 593-599.

35. Fujimoto T, Budgell B, Uchida S, Suzuki A, Meguro K (1999) Arteria tonometry in the measurement of the effects of innocuous mechanical stimulation of the neck on heart rate and blood pressure. J Auton Nery Syst 75: 109-115.

36. Carrick FR (1997) Changes in brain function after manipulation of the cervical spine. J Manipulative Physiol Ther 20: 529-545.

37. Roberts S, Eisenstein SM, Menage J, Evans EH, Ashton IK (1995) Mechanoreceptors in intervertebral discs. Morphology, distribution, and neuropeptides. Spine (Phila Pa 1976) 20: 2645-2651.

38. Revel M, Minguet M, Gregoy P, Vaillant J, Manuel JL (1994) Changes in cervicocephalic kinesthesia after a proprioceptive rehabilitation program in patients with neck pain: a randomized controlled study. Arch Phys Med Rehabil 75: 895-899.
39. Kruse MN, Mallory BS, Noto H, Roppolo JR, de Groat WC (1991) Properties of the descending limb of the spinobulbospinal micturition reflex pathway in the cat. Brain Res 556: 6-12.

40. Haavik H, Niazi IK, Jochumsen M, Sherwin D, Flavel S, et al. (2016) Impact of Spinal Manipulation on Cortical Drive to Upper and Lower Limb Muscles. Brain Sci 7.

41. Taylor HH, Holt K, Murphy B (2010) Exploring the Neuromodulatory Effects of the Vertebral Subluxation and Chiropractic Care. Chiropr J Aust 40: $37-44$

42. Lelic D, Niazi IK, Holt K, Jochumsen M, Dremstrup K, et al. (2016) Manipulation of Dysfunctional Spinal Joints Affects Sensorimotor Integration in the Prefrontal Cortex: A Brain Source Localization Study. Neural Plast 3704964

43. de Almeida BS, Sabatino JH, Giraldo PC (2010) Effects of high-velocity, low-amplitude spinal manipulation on strength and the basal tonus of female pelvic floor muscles. J Manipulative Physiol Ther 33: 109-116.

44. Taylor HH, Murphy B (2008) Altered Sensorimotor Integration with Cervical Spine Manipulation. J Manipulative Physiol Ther 31: 115-126. 